\title{
Torsión tubárica aislada como complicación de un quiste paraovárico en el Hospital Universitario de Santander, Colombia
}

\author{
Isolated Fallopian Tube Torsion as a Complication of a Paraovarian \\ Cyst in the University Hospital of Santander, Colombia
}

\section{Torção tubária isolada como uma complicação de um cisto paraovariano no Hospital Universitário de Santander, Colômbia}

\author{
Janer Sepúlveda-Agudelo, MD., Esp. * \\ Daniel Mauricio Torrado-Arenas, MD. ** \\ Nathalia Martínez-Moreno, MD. ***
}

\section{Resumen}

Introducción: El quiste paraovárico representa el 10 al 20\% de las masas anexiales, genera síntomas cuando causa torsión de los anexos, pero es poco frecuente, la torsión aislada de las trompas tiene una incidencia de 1 en 1.5 millones de mujeres. Objetivo: Presentar un caso de torsión tubárica aislada como complicación de un quiste paraovárico en el Hospital Universitario de Santander. Caso clínico: Paciente femenino de 17 años, presenta dolor en hemiabdomen inferior; hallazgo ecográfico, quiste paraovárico derecho; mejora la sintomatología en 24 horas; egreso con recomendaciones; regresa dos meses después por exacerbación del dolor, masa anexial de 5 centímetros, laboratorios normales, ecografía reporta ovario aumentado de tamaño y trompa de paredes engrosadas; se realiza laparoscopia; se encuentra trompa derecha torcida con necrosis y quiste paraovárico derecho de 6 x $5 \mathrm{~cm}$. Discusión: El diagnóstico de quiste paraovárico es generalmente incidental por ecografía, cuando se complica con torsión, se presenta dolor abdominal pélvico asociado a náuseas y vómito, al examen físico el $42.9 \%$ de las mujeres presentan a la palpación una masa anexial; la paciente del caso presenta dolor abdominal, con masa anexial palpable evidenciada en ecografía, por lo cual se realiza laparoscopia. El tratamiento del quiste paraovárico es el seguimiento, sin embargo, si aumentan de tamaño o hay persistencia, debe retirarse quirúrgicamente por el riesgo de torsión de anexos. De presentar torsión el manejo depende del estado de la trompa, al realizar destorsión persiste la coloración violácea, se debe realizar salpingectomía y cistectomía procedimiento realizado en esta paciente. Conclusiones: El diagnóstico de la torsión tubárica se realiza con clínica de dolor abdominal pélvico agudo, náuseas, vómito y presencia de masa anexial al tacto vaginal. La laparoscopia es el tratamiento de elección, cuyo manejo con cistectomía y salpingectomía que depende del compromiso vascular de los anexos. [Sepúlveda-Agudelo J, Torrado-Arenas DM, Martínez-Moreno N. Torsión tubárica aislada como complicación de un quiste paraovárico en el Hospital Universitario de Santander, Colombia. MedUNAB 2017; 20(3): 393-398].

Palabras clave: Quiste Paraovárico; Anomalía Torsional; Laparoscopia; Trompas Uterinas; Ultrasonografía.

* Médico, especialista en Ginecología y Obstetricia, subespecialista en Cirugía Endoscópica Ginecológica, profesor asociado al Departamento de Ginecología y Obstetricia, Universidad Industrial de Santander, Bucaramanga, Santander, Colombia.

** Médico y Cirujano, Universidad Industrial de Santander, miembro del grupo de investigación GINO (Ginecología-UIS), Bucaramanga, Santander, Colombia.

*** Médico y Cirujano, Universidad Industrial de Santander, Bucaramanga, Santander, Colombia.

Correspondencia: Janer Sepúlveda Agudelo. Calle 49 No. 28-10, consultorio 302, Bucaramanga, Santander, Colombia. Teléfono: +57 3153756341. E-mail: janersepulveda@yahoo.es 


\section{Abstract}

Introduction: The paraovarian cyst represents from $10 \%$ to $20 \%$ of the adnexal masses, it generates symptoms when it causes torsion of the annexes but this is rare. The isolated torsion of the fallopian tubes has an incidence of 1 in 1.5 million women. Objective: To show a case of isolated fallopian tube torsion (IFTT) as a complication of a paraovarian cyst at the University Hospital of Santander. Clinical case: A 17-year old female patient who presents pain in lower hemi-abdomen. After performing the ultrasound, a right paraovarian cyst is found. Her symptomatology improves within 24 hours. Therefore, she is discharged after telling her some recommendations. Two months later, she returns because of pain exacerbation caused by an adnexal mass of 5 centimeters. Now, her lab tests are normal but her new ultrasound reports an enlargement in the ovary and thickened fallopian tubes, so a laparoscopy is performed. This test shows that the right fallopian tube is twisted with necrosis and a right paraovarian cyst of $6 \times 5 \mathrm{~cm}$. Discussion: The diagnosis of paraovarian cyst is usually incidental by ultrasound; but when there is a torsion complication, pelvic abdominal pain associated with nausea and vomiting is presented. When the physical examination is performed, $42.9 \%$ of women present an adnexal mass on palpation. The patient of this clinical case shows abdominal pain, including a tangible adnexal mass, which is evidenced by the ultrasound. For this reason, a laparoscopy is performed. The treatment of the paraovarian cyst is the follow-up; however, if it increases in size or there is persistence, it should be removed surgically due to the risk of torsion of its annexes. If it presents torsion, its handling will depend on the state of the tube. So, if at the moment of performing a distortion in it the violaceous color persists, a salpingectomy and a cystectomy must be performed in this patient. Conclusions: The diagnosis of fallopian tube torsion is performed if the patient has clinical symptoms such as acute pelvic abdominal pain, nausea, vomiting and presence of adnexal mass when performing a digital vaginal examination. Laparoscopy is the treatment of choice, and its management with cystectomy and salpingectomy depends on the vascular commitment of the cyst annexes. [Sepúlveda-Agudelo J, Torrado-Arenas DM, Martínez-Moreno N. Isolated Fallopian Tube Torsion as a Complication of a Paraovarian Cyst in the University Hospital of Santander, Colombia. MedUNAB 2017; 20(3): 393-398].

Key words: Paraovarian Cyst; Torsion Abnormality; Laparoscopy; Fallopian Tubes; Ultrasonography.

\section{Introduction}

El quiste paraovárico o paratubárico representa del $10 \%$ al $20 \%$ de las masas anexiales, su hallazgo es más común entre la tercera y cuarta década de la vida (1). El quiste paraovárico se evidencia en pacientes asintomáticas, lo que hace más difícil su diagnóstico, se presentan síntomas cuando hay ruptura, torsión o cuando el quiste supera los 5.0 centímetros (cm) de diámetro (1-3); su hallazgo habitualmente es incidental; se ha reportado torsión anexial de la trompa uterina, del ovario o de ambas estructuras, siendo más frecuente el segundo caso (4). La incidencia no

\section{Resumo}

Introdução: o cisto paraovariano representa 10 a $20 \%$ das massas anexiais, gera sintomas quando provoca a torção dos anexos, mas é raro, a torção isolada dos tubos tem uma incidência de 1 em 1,5 milhão de mulheres. Objetivo: Apresentar um caso de torção tubária isolada como complicação de um cisto paraovariano no Hospital Universitário de Santander. Caso clínico: paciente do sexo feminino de 17 anos, apresenta dor em hemiabdomen inferior; com ultra-som foi descoberto um cisto paraovariano direito; no prazo de 24 horas, melhora a sintomatologia; sai de alta, com recomendações. Retorna dois meses depois, por causa da dor intense e uma massa anexial de 5 centímetros $(\mathrm{cm})$, como resultado nos laboratórios normais. O ultra-som revelou o aumento do tamanho do ovário e o bico afundado; é realizada uma laparoscopia; O tronco torcido direito é encontrado com necrose e cisto paraovariano direito de $6 \times 5 \mathrm{~cm}$. Discussão: $O$ diagnóstico do cisto paraovariano geralmente é descoberto por incidente, ao se fazer a ultrassonografia, quando é um caso complicado com torção, a dor abdominal pélvica está associada a náuseas e vômitos; no exame físico, 42,9\% das mulheres apresentam palpação, uma massa anexial. A paciente, no caso apresenta dor abdominal, com uma massa anexial palpável evidenciada no ultra-som, razão pela qual a laparoscopia é realizada. O tratamento do cisto paraovariano é o acompanhamento, no entanto, se eles aumentam de tamanho ou haja persistência, ele deve ser removido cirurgicamente devido ao risco de torção de anexos. Se a torção persiste, o tratamento depende do estado do tubo e, se ao realizar a distorção, a cor violácea persiste, a salpingectomia e a cistectomia devem ser realizadas nessa paciente. Conclusões: o diagnóstico de torção tubária é feito com sintomas de dor abdominal pélvica aguda, náuseas, vômitos e presença de massa anexial ao toque vaginal. A laparoscopia é o tratamento de escolha, cujo manejo com cistectomia e salpingectomia depende do comprometimento vascular dos anexos. [SepúlvedaAgudelo J, Torrado-Arenas DM, Martínez-Moreno N. Torção tubária isolada como uma complicação de um cisto paraovariano no Hospital Universitário de Santander, Colômbia. MedUNAB 2017; 20(3): 393-398].

Palavras-chave: Cisto Parovariano; Anormalidade Torcional; Laparoscopia; Tubas Uterinas; Ultrassonografia.

es claramente conocida, pero en una población italiana se reportó una incidencia de 29 por 1000 (3\%), sin embargo, en población pediátrica es reportada con una incidencia más alta que alcanza el 7.3\% (4), no hay reportes de incidencia en Colombia de esta patología.

La torsión anexial abarca aproximadamente el 3.0\% de las urgencias ginecológicas (5), cuya fisiopatología es dada por la obstrucción arterial y venosa debido a la rotación de una o todas las estructuras anexiales, lo cual lleva a necrosis que puede terminar en peritonitis y sepsis $(6,7)$; por otra parte, la torsión aislada de la tuba uterina tiene una incidencia de 1.0 caso por cada 1.5 millones de mujeres (7-9). La trompa 
derecha tiene tres veces más riesgo de sufrir torsión, debido a las diferencias en el retorno venoso, la amplia congestión en el lado derecho y la posición estable que le brinda el sigmoides, parcialmente adherido a la trompa izquierda (4, 10). En general, la torsión de los anexos por un quiste paraovárico debe considerarse como diagnóstico diferencial en mujeres con dolor agudo en hemiabdomen inferior con masa anexial, independientemente de la edad (7, 11, 12); debido a que hay casos reportados en pacientes premenárquicas y post-menopaúsicas (7); por otro lado, la posibilidad de malignidad es rara, se han publicado como reporte de casos en la literatura, como quistes paratubáricos en el límite de malignidad(13).

Con base en lo anterior, se considera de interés la presentación del caso, porque debe incluirse entre los diagnósticos diferenciales de dolor abdominal bajo, de aparición aguda, en mujeres a cualquier edad; muchas veces es confundido con otras patologías como apendicitis, ruptura de un quiste ovárico, que tienden a ser más frecuentes; como antecedentes ya se han reportado casos similares en diferentes contextos de la literatura mundial; resaltando el manejo terapéutico, desde el abordaje con laparotomía hasta el uso menos invasivo como la laparoscopia $(1,2,14-16)$.

El objetivo es presentar el primer caso reportado de quiste paraovárico con torsión de la trompa sin compromiso del ovario, ocurrido en el Hospital Universitario de Santander (HUS) con previo consentimiento informado. Este caso aporta a la literatura que ante mujeres con dolor pélvico agudo con palpación de masa anexial, la torsión tubárica debe descartase, donde la laparoscopía es una herramienta útil al ser diagnóstica y terapéutica.

\section{Caso Clínico}

Paciente femenina de 17 años de edad, G0P0, procedente de Bucaramanga, estudiante de secundaria, que consulta por dolor intenso en fosa iliaca derecha, tipo cólico de intensidad 8 sobre 10, en escala análoga del dolor, irradiado a región lumbar y miembro inferior derecho, de 4 horas de evolución, acompañado de retardo menstrual de un mes; afebrily sin otros síntomas asociados.

Antecedentes personales negativos, historia ginecoobstétrica: menarquia a los 14 años, ciclos menstruales irregulares e inicio de vida sexual a los 15 años, planificación con inyectables combinados, hasta el mes anterior de consulta; no refería enfermedades de transmisión sexual, última citología normal realizada hace un año, no fumadora, negativo para alergias; ningún antecedente patológico, quirúrgico y familiar de importancia. Inicialmente en el examen físico se encontró abdomen blando, depresible, con dolor a la palpación en fosa iliaca derecha, sin signos de irritación peritoneal; al tacto vaginal cuello posterior, largo, cerrado, sin lesiones; en anexo derecho se palpó una masa aproximadamente de $5.0 \mathrm{~cm}$, no se evidenció sangrado, ni leucorrea.

Ecografía transvaginal, masa hipoecoica de 59.9 × 45.7 x 57.6 milímetros, compatible con quiste anexial derecho, hormona gonadotropina coriónica subunidad Beta cuantificada (Beta HCG) negativa, cuadro hemático, proteína $\mathrm{C}$ reactiva y parcial de orina normales; se decide hospitalizar, mejora con líquidos endovenosos, antiespasmódicos y reposo; a las 24 horas cesó el dolor abdominal, se da egreso, explicándole signos de alarma y control por consulta externa en ginecología laparoscópica, para manejo del quiste paraovárico ambulatorio. Por otro lado, la paciente persiste con dolor intermitente y regresa dos meses después por exacerbación del dolor abdominal. En el nuevo examen físico se halló álgica con signos vitales normales, dolor a la palpación en hemiabdomen inferior, puño percusión derecha positiva, leucorrea grumosa, persistencia de masa anexial y sin signos de irritación peritoneal.

Posteriormente se realizó ecografía transvaginal, evidenciando por segunda vez el quiste anexial derecho con tuba uterina de paredes gruesas. Los exámenes paraclínicos mostraron un cuadro hemático y parcial de orina normales, Beta HCG negativa, proteína $\mathrm{C}$ reactiva en $12.0 \mathrm{mg} / \mathrm{l}$. Se hospitalizó, a 14 horas de su ingreso se consideró llevar a cirugía laparoscópica diagnóstica con previo consentimiento informado, para descartar una posible torsión ovárica. El procedimiento fue llevado sin ninguna complicación quirúrgica o anestésica; se encontró trompa derecha de color violáceo, con cinco vueltas y un quiste paraovárico derecho aproximadamente de 6.0 x $5.0 \mathrm{~cm}$, necrosado; mientras los ovarios, trompa izquierda y útero se observaron normales; se realizó salpingectomía derecha más resección de quiste paraovárico derecho, utilizando energía bipolar y corte con tijera; el material extraído se envió a patología; la paciente se mantuvo en recuperación con analgesia, estable hemodinámicamente y se dio egreso el mismo día con cita de control.

Al control post operatorio a los ocho días del procedimiento; se encontró asintomática, afebril, sin dolor abdominal, con herida quirúrgica sin signos de infección. El reporte de patología reveló un diagnóstico de quiste simple mesotelial con cambios de infarto hemorrágico, trompa uterina con congestión vascular y necrosis, sin malignidad (Figura 1, 2, 3). La paciente manifestó una experiencia satisfactoria con el uso de laparoscopia y a la evolución clínica.

\section{Discusión}

Los quistes paraováricos son unas estructuras en forma de bolsa que se forman entre el mesosalpinx del ligamento ancho entre el ovario y la trompa uterina por estímulo 


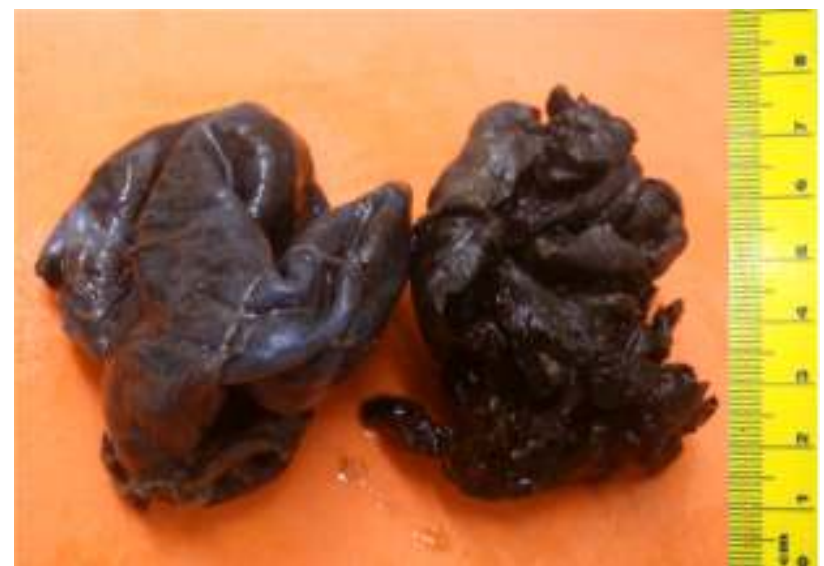

Fuente: Autores

Figura 1. Imagen macroscópica de quiste paraovárico derecho en preparación de formol.

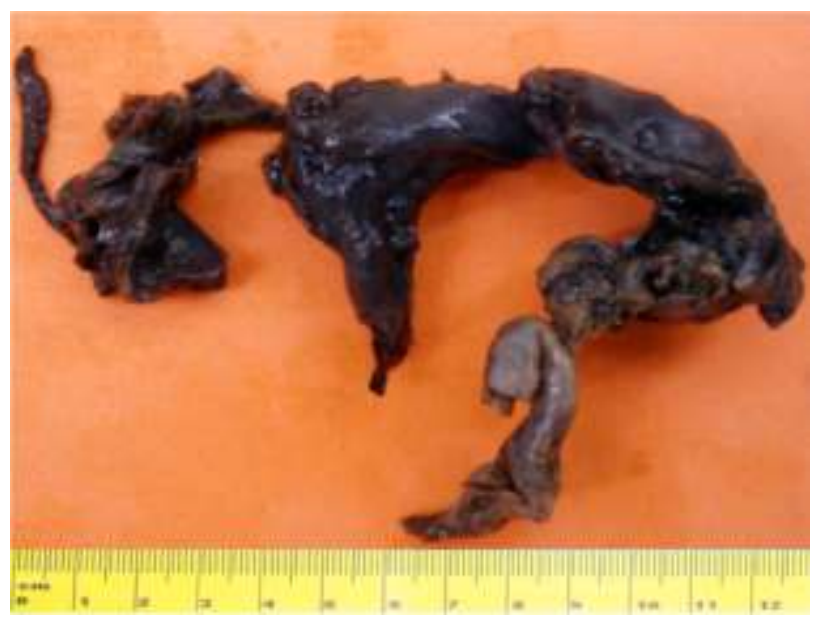

Fuente: Autores.

Figura 2. Imagen macroscópica de trompa uterina con infarto en preparación de formol.

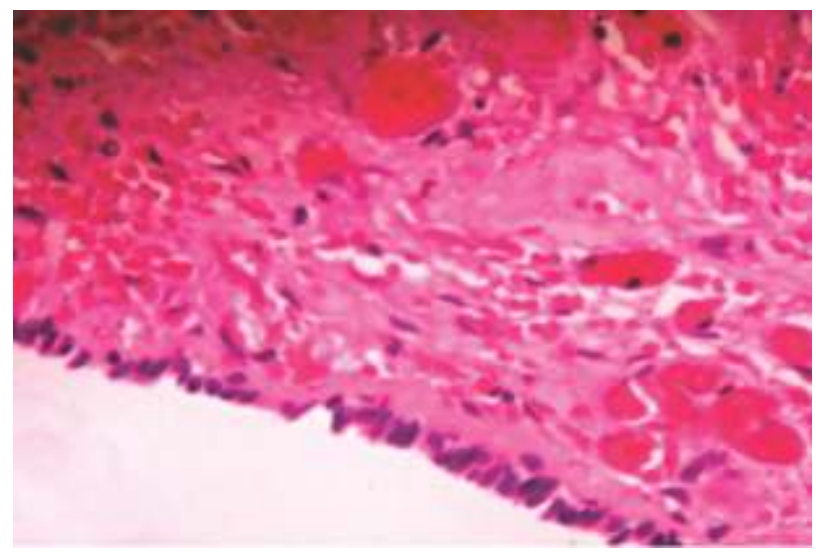

Fuente: Autores

Figura 3. Imagen microscópica de epitelio cúbico simple que reviste la superficie interna del quiste simple. Se descartó malignidad. hormonal (14). Muolokwu y colaboradores, encontraron en una serie de casos que el tamaño de estos quistes varía de 0.5 a $33.0 \mathrm{~cm}$, con un promedio de $6.13+/-5.9 \mathrm{~cm}(16)$, esta medida promedio coincide con este caso, que presentaba un tamaño de $6 \mathrm{~cm}$. Por otro lado, vale la pena resaltar que si el quiste paraovárico es pediculado y está localizado cerca de las fimbrias de la trompa, se le denomina quiste hidatídico de Morgagni, el cual usualmente tiene un diámetro menor de $2.0 \mathrm{~cm}(14)$

Los hallazgos ecográficos del quiste paraovárico son una masa quística, redonda u oval, bien definida, de pared delgada, ubicada proximal al ovario ipsilateral, manteniendo un espacio pequeño frente a este (17). Puede hallarse el signo de división (Split sign), consiste en empujar ligeramente con el transductor transvaginal el abdomen de la paciente y observar movimientos oscilatorios opuestos entre el ovario y el quiste (18). No obstante, Barloon y colaboradores, refieren que estos quistes son difíciles de diagnosticar con ultrasonografía antes de la cirugía y el diagnóstico prequirúrgico sólo es posible en el 6.6\% de los casos (19); en este caso se informó como quiste anexial derecho, sin precisar que se trataba de un quiste paraovárico (Figura 4).

Su estudio histopatológico revela diferentes orígenes, en el trabajo de Genadry se analizaron 132 quistes paraováricos, encontrando que el $68.0 \%$ correspondían a mesotelio, el $30.0 \%$ a derivados paramesonéfricos y el $2.0 \%$ a restos mesonéfricos (1). La mayoría de este tipo quistes son benignos, la incidencia reportada de malignidad es del $2.0 \%$ al 3.0\% (18); una de sus características es el hallazgo de proyecciones papilares en la pared interna del quiste (14, 20). Los hallazgos patológicos del presente caso revelaron un quiste simple, el cual concuerda con la clasificación de origen mesotelial, que es la más frecuente.

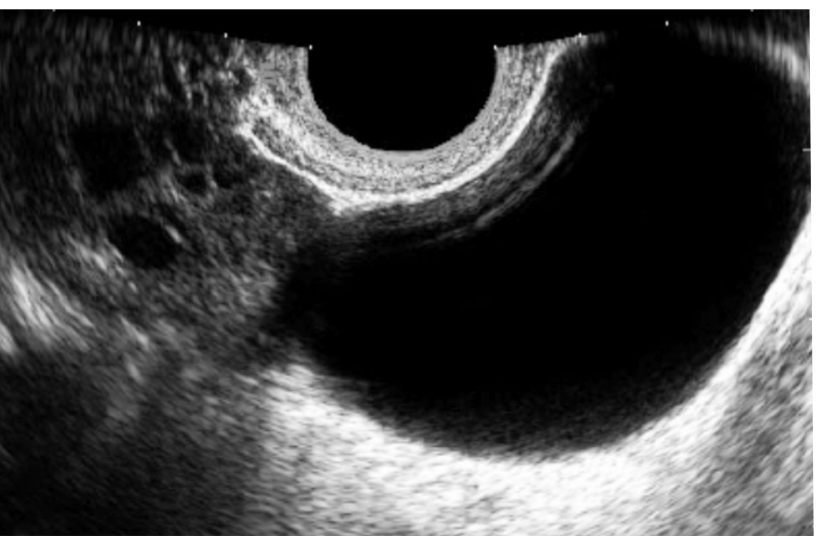

Fuente: Savelli L, Ghi T, De laco P, Ceccaroni M, Venturoli S, Cacciatore B. Paraovarian/paratubal cysts: comparison of transvaginal sonographic and pathological findings to establish diagnostic criteria. Ultrasound Obstet Gynecol. 2006; 28(3): 330-4. Disponible en: https://doi.org/10.1002/uog.2829.

Figura 4. Imagen ecográfica de quiste paraovárico derecho proximal al ovario ipsilateral, manteniendo un espacio pequeño frente a este. 
El diagnóstico de quiste paraovárico es generalmente incidental por ecografía, sin embargo, cuando se complica con torsión de los anexos ipsilateral comienza a generar sintomatología; la presentación más frecuente es dolor abdominal pélvico, asociado a náuseas y vómito, con un $87.1 \%$ y $65.7 \%$ de los casos respectivamente; se debe tener en cuenta que la paciente adicionalmente puede tener síntomas inespecíficos de tipo gastrointestinal y urinario (5). Al examen físico, menos de la mitad de las mujeres tienen a la palpación una masa anexial; Nair y colaboradores reportan $42.9 \%$ de las pacientes tenían masa al tacto vaginal (5). Además de la clínica en la torsión anexial, se puede usar ecografía con una sensibilidad del 40.0 al $75.0 \%$, cuyos hallazgos incluyen: trompa dilatada, trenzada, con paredes gruesas ecogénicas y contenido hemorrágico $(5,14)$. Otros autores reportan el uso de la tomografía axial computarizada, donde se observa la trompa dilatada e hipodensa, útero desviado de forma ipsilateral (8). Sin embargo, ninguna de las anteriores ayudas imagenológicas tiene criterios específicos para el diagnóstico (14). La paciente del caso no tiene ningún antecedente ginecológico y su sintomatología solamente es dolor abdominal en fosa iliaca derecha, con masa anexial al tacto vaginal, cuyos paraclínicos eran normales, solamente la ecografía muestra algunos hallazgos incipientes, por lo cual la laparoscopia se usa ante la duda con posibilidad de otros diagnósticos diferenciales en mujer en edad fértil.

El tratamiento del quiste paraovárico es el seguimiento, sin embargo, si aumentan de tamaño considerablemente o si hay persistencia debe retirarse quirúrgicamente por el riesgo de torsión de anexos (16). No obstante, si termina en este último escenario, la laparoscopia es el procedimiento de elección o Gold estándar, debido a que es diagnóstica y terapéutica (21) en dolor abdominal quirúrgico con masa anexial, además el retorno a las actividades normales es más rápido, al igual que el tiempo de estancia hospitalaria y la pérdida sanguínea es menor comparada con la laparotomía $(4,8,22,23)$. El manejo de la torsión depende del compromiso vascular, estado de la trompa en el acto quirúrgico, si posterior a la destorsión persiste la coloración violácea y el tiempo de evolución es largo por la necrosis se debe realizar salpingectomía y cistectomía. Sin embargo, con el fin de conservar la fertilidad, si hay vitalidad de la trompa, se realiza sólo la cistectomía con destorsión y fijación de tuba para prevenir recurrencias (5); la extirpación del ovario ipsilateral no está indicada en aquellos casos de torsión tubárica aislada (5); en este caso, por ser una torsión aislada se conservó el ovario como lo recomienda la literatura.

Es importante anotar que existen factores intrínsecos y extrínsecos para la etiología de la torsión, dentro de los primeros, que están relacionados directamente con la misma trompa, se incluye una gran longitud de la tuba uterina e hidrosalpinx y los segundos son los atribuibles al trauma, las masas anexiales, como el presente caso descrito
$(9,10)$. La incidencia de torsión de la trompa en las pacientes con quistes paraováricos oscila entre el $2.1 \%$ al $16.0 \%(14,24)$; lo cual concuerda con el estudio retrospectivo de Nair y colaboradores, donde un total de 70 pacientes con torsión anexial que consultaron a urgencias, $14.3 \%$ tenían como causa un quiste paraovárico $(5,25)$; actualmente, se desconoce la incidencia en nuestro país.

Finalmente, llama la atención en esta paciente que se descartaron las posibilidades diagnósticas más frecuentes como embarazo ectópico con la Beta HCG negativa, y la apendicitis con unos reactantes de fase aguda negativos y un cuadro hemático normal. Sin embargo, de este caso hay limitaciones como la falta de imágenes ecográficas de la lesión, que no fueron tomadas durante la realización del examen. Las similitudes con otras patologías que producen dolor abdominal dificultan su diagnóstico, retrasa su manejo quirúrgico que tiene consecuencias en la fertilidad de la mujer $(10,19)$. Por lo cual ante esta patología lo más importante es el diagnóstico y detección temprana, para no comprometer la fertilidad de la mujer (8).

\section{Conclusiones}

La torsión de la trompa, a pesar de ser menos frecuente que patologías como apendicitis, debe incluirse entre los diagnósticos diferenciales de dolor abdominal bajo, de aparición aguda, con o sin masa anexial en mujeres independientemente de la edad. Por otro lado, la torsión de la trompa uterina como complicación de un quiste paraovárico es de difícil diagnóstico prequirúrgico; por lo cual es importante realizar una adecuada historia clínica, tener en cuenta los hallazgos ecográficos y usar la laparoscopia al ser diagnóstica y terapéutica. Finalmente, en el manejo de la torsión lo ideal es hacer el diagnóstico y el manejo temprano, para así evitar el compromiso de la fertilidad futura de la mujer, donde la laparoscopia es el Gold estándar para manejo del quiste paraovárico y sus complicaciones.

\section{Responsabilidades éticas}

Protección de personas y animales: Los autores declaran que para esta investigación no se han realizado experimentos en seres humanos ni en animales.

Confidencialidad de los datos: Los autores declaran que han seguido los protocolos de su centro de trabajo sobre la publicación de datos de pacientes.

Derecho a la privacidad y consentimiento informado: Los autores han obtenido el consentimiento y asentimiento informado de la paciente referida en el artículo. Este documento obra en poder del autor de correspondencia. 


\section{Conflicto de intereses}

Los autores manifiestan que no tienen ningún conflicto de interés.

\section{Referencias}

1. Hasuo $Y$, Higashijima T, Mitamura T. Torsion of paraovarian cyst. Report of two cases. Kurume Med J. 1991; 38(1):39-43.

2. Said MR, Bamigboye V. Twisted paraovarian cyst in a young girl. J Obstet Gynaecol 2008; 28(5): 549-50. Disponible en: https://doi.org/10.1080/ 01443610802247444

3. Grover S. Torsion causing interruption of the ampullary portion of the fallopian tube. Fertil Steril. 2007; 88(4): 968.e13-4. Disponible en: https://doi.org/10.1016/ j.fertnstert.2006.11.159.

4. Dadhwal V, Gupta N, Gupta B, Deka D, Mittal S. Laparoscopic management of isolated fallopian tube torsion in a premenarchal 13-year-old adolescent girl. Arch Gynecol Obstet. 2009; 279(6): 909-10. Disponible en: https://doi.org/10.1007/s00404-008-0809-y.

5. Nair S, Joy S, Nayar J. Five Year Retrospective Case Series of Adnexal Torsion. J Clin Diagn Res. 2014; 8(12): 9-13. Disponible en: https://doi.org/10.7860/JCDR/ 2014/9464.5251.

6. Ferrera PC, Kass LE, Verdile VP. Torsion of the Fallopian Tube. Am J Emerg Med. 1995; 13(3): 312-4. Disponible en: https://doi.org/10.1016/07356757(95)90208-2.

7. Zanden M, Nap A, Kints $M$. Isolated torsion of the fallopian tube: a case report and review of the literature. Eur J Pediatr. 2011; 170(10): 1329-32. Disponible en: https://doi.org/10.1007/s00431-011-1484-8.

8. Rangasamy A, Ramalingam M, Crichton F. P953 Fallopian tube torsion: a case series. The role of paratubal cysts. Int J Gynecol Obstet. 2009, 107: S681. Disponible en: https://doi.org/10.1016/S00207292(09)62440-1.

9. Bodega A, Alario I, Crespo M, González M, Izquierdo M, Díaz-Miguel V. Torsión aislada de la trompa de Falopio. Tratamiento endoscópico. Clin Invest Gin Obst. 2004; 31(8): 302-4. Disponible en: https://doi.org/ 10.1016/S0210-573X(04)77353-0.

10. Ledesma I, Castañón L, Álvarez R, Herrero B, Orille V. Torsión aislada de la trompa de Falopio en una niña premenárquica. Bol Pediatr. 2005; 45: 33-6. Disponible en: https://sccalp.org/documents/0000/1027/ BolPediatr2005_45_033-036.pdf.

11. Uret E, Blanco A. Quiste paraovárico complicado: causa rara de dolor abdominal. Rev. chil. Radiol. 2007; 13(3): 159-162. Disponible en: https://doi.org/10.4067/S071793082007000300008 .

12. Comerci G, Colombo FM, Stefanetti M, Grazia G. Isolated fallopian tube torsion: a rare but important event for women of reproductive age. Fertil Steril. 2008; 90(4):1198.e23-e25. Disponible en: https://doi.org/ 10.1016/j.fertnstert.2007.08.057.

13. Terek MC, Sahin C, Yeniel AO, Ergenoglu M, Zekioglu O. Paratubal borderline tumor diagnosed in the adolescent period: a case report and review of the literature. J
Pediatr Adolesc Gynecol. 2011; 24(5):115-6. Disponible en: https://doi.org/10.1016/j.jpag.2011.05.007.

14. Kiseli M, Caglar GS, Cengiz SD, Karadag D, Yilmaz MB. Clinical diagnosis and complications of paratubal cysts: review of the literature and report of uncommon presentations. Arch Gynecol Obstet. 2012; 285(6):15639. Disponible en: https://doi.org/10.1007/s00404-0122304-8.

15. Macarthur M, Mahomed A. Laparoscopy in the diagnosis and management of a complicated paraovarian cyst. SurgEndosc. 2003; 17(10): 1676-7. Disponible en: https://doi.org/10.1007/s00464-003-4211-3.

16. Muolokwu E, Sanchez J, Bercaw JL, Sangi-Haghpeykar $H$, Banszek T, Brandt $M L$, et al. The incidence and surgical management of paratubal cysts in a pediatric and adolescent population. J Pediatr Surg. 2011; 46(11): 2161-3. Disponible en: https://doi.org/10.1016/ j.jpedsurg.2011.04.054.

17. Harmon JC, Binkovitz LA, Binkovitz LE. Isolated fallopian tube torsion: sonographic and CT features. Pediatr Radiol. 2008; 38(3):175-9. Disponible en: https://doi.org/10.1007/s00247-007-0683-y.

18. Savelli L, Ghi T, De laco P, Ceccaroni M, Venturoli S, Cacciatore B. Paraovarian/paratubal cysts: comparison of transvaginal sonographic and pathological findings to establish diagnostic criteria. Ultrasound Obstet Gynecol. 2006; 28(3): 330-4. Disponible en: https://doi.org/ 10.1002/uog.2829.

19. Barloon TJ, Brown BP, Abu-Yousef MM, Warnock NG. Paraovarian and paratubal cysts: preoperative diagnosis using transabdominal and transvaginalsonography. $\mathrm{J}$ Clin Ultrasound. 1996; 24(3): 117-22. Disponible en: h t t p s: // doi.org/10.1002/( S I C I) 1097 . 0096(199603)24:3<117::AID-JCU2>3.0.CO;2-K.

20. Shin YJ, Kim JY, Lee HJ, Park JY, Nam JH. Paratubal serous borderline tumor. J Gynecol Oncol. 2011; 22(4): 295-8. Disponible en: https://doi.org/10.3802/ jgo.2011.22.4.295.

21. Leanza V, Coco L, Genovese F, Pafuml C, Ciotta L, Leanza G, et al. Laparoscopic removal of a giant paratubal cyst complicated by hydronephrosis. G Chir. 2013; 34(11-12): 323-325. Disponible en: https://www.ncbi.nlm.nih.gov/pmc/articles/PMC392647 2/?report=reader

22. Smith AL, Bieber EJ. The Diagnostic Challenge of Identifying Isolated Fallopian Tube Torsion: A Case Report of Laparoscopic Management. J Minim Invasive Gynecol. 2008; 15(4): 514-6. Disponible en: https://doi.org/10.1016/j.jmig.2008.04.013.

23. Torres J, Íñiguez R. Quiste paraovárico gigante en la infancia. Reporte de un caso. Rev chil pediatr. 2015; 86 ( 2): $117-120$. D i s p o n i ble e n : https://doi.org/10.1016/j.rchipe.2015.04.023.

24. Ardıçlı B, EkinciS, Oğuz B, Haliloğlu M, Tanyel C, Karnak I. Laparoscopic detorsion of isolated idiopathic Fallopian tuve torsion: conservative treatment in a 13-year-old girl. Turk J Pediatr. 2013; 55:451-4. Disponible en: https://pdfs.semanticscholar.org/b70b/674651a030b2a 238039e669e720f9572a885.pdf

25. Gedam JK, Rajput DA, Bhalerao MV. Torsion-of paraovarian cyst resulting in secondary torsion of the fallopian tube: a cause of acute abdomen. J Clin Diagn Res. $2014 ; 8(5): 10-1$. Disponible en: https://doi.org/10.7860/JCDR/2014/7946.4386. 